PROCEEDINGS OF THE

AMERICAN MATHEMATICAL SOCIETY

Volume 137, Number 1, January 2009, Pages 211-218

S 0002-9939(08)09488-4

Article electronically published on April 25, 2008

\title{
A SPECTRAL EXCLUSION PRINCIPLE FOR UNBOUNDED SUBNORMALS
}

\author{
SAMEER CHAVAN
}

(Communicated by N. Tomczak-Jaegermann)

\begin{abstract}
We establish a Spectral Exclusion Principle for unbounded subnormals. As an application, we obtain some polynomial approximation results in the functional model spaces.
\end{abstract}

\section{Preliminaries}

For a subset $A$ of the complex plane $\mathcal{C}$, let $A^{*}, \operatorname{int}(A), \bar{A}$ and $A^{c}$ respectively denote the conjugate, the interior, the closure and the complement of $A$ in $\mathcal{C}$. We use $\mathcal{R}$ to denote the real line, and $\operatorname{Re} z$ and $\operatorname{Im} z$ respectively denote the real and imaginary parts of a complex number $z$. Let $\mathcal{H}$ be a complex infinite-dimensional separable Hilbert space with the inner product $\langle\cdot, \cdot\rangle_{\mathcal{H}}$ and the corresponding norm $\|\cdot\|_{\mathcal{H}}$. If $S$ is a densely defined linear operator in $\mathcal{H}$ with domain $\mathcal{D}(S)$, then we use $\sigma(S), \sigma_{p}(S), \sigma_{a p}(S)$ to respectively denote the spectrum, the point spectrum and the approximate point spectrum of $S$. It may be recalled that $\sigma_{p}(S)$ is the set of eigenvalues of $S$, that $\sigma_{a p}(S)$ is the set of those $\lambda$ in $\mathcal{C}$ for which $S-\lambda$ is not bounded below, and that $\sigma(S)$ is the complement of the set of those $\lambda$ in $\mathcal{C}$ for which $(T-\lambda)^{-1}$ exists as a bounded linear operator on $\mathcal{H}$. For a normal operator $N$ in $\mathcal{H}, \sigma(N)=\sigma_{a p}(N)$. For a non-negative measure $\mu$ on the complex plane $\mathcal{C}$, we will use $\operatorname{supp}(\mu)$ to denote the support of $\mu$.

In the present section, we record a few requisites pertaining to the unbounded subnormals and the $m$ - $\Sigma$-accretive operators. In Section 2, we prove a Spectral Exclusion Principle for unbounded subnormals. We use this principle to obtain $H^{\infty}$ functional calculi for unbounded subnormals. As an application, we present some polynomial approximation results in the functional model spaces. These results rely heavily on the results of Crouzeix and Delyon regarding the $m$ - $\Sigma$-accretive operators (4], Chapter 7).

1.1. Unbounded subnormals. A densely defined linear operator $S$ in $\mathcal{H}$ with domain $\mathcal{D}(S)$ is said to be cyclic if there is a vector $f_{0} \in \mathcal{D}^{\infty}(S) \equiv \bigcap_{n=0}^{\infty} \mathcal{D}\left(S^{n}\right)$ (referred to as a cyclic vector of $S$ ) such that $\mathcal{D}(S)$ is the linear span $\operatorname{lin}\left\{S^{n} f_{0}\right.$ : $n \geq 0\}$ of the set $\left\{S^{n} f_{0}: n \geq 0\right\}$.

If $S$ is a densely defined linear operator in $\mathcal{H}$ with domain $\mathcal{D}(S)$, then $S$ is said to be subnormal if there exist a Hilbert space $\mathcal{K}$ containing $\mathcal{H}$ and a densely defined

Received by the editors April 26, 2007, and, in revised form, December 23, 2007.

2000 Mathematics Subject Classification. Primary 47A60, 47B20; Secondary 41A10.

Key words and phrases. Unbounded subnormal, $H^{\infty}$ functional calculus.

(C)2008 American Mathematical Society Reverts to public domain 28 years from publication 
normal operator $N$ in $\mathcal{K}$ with domain $\mathcal{D}(N)$ such that $\mathcal{D}(S) \subset \mathcal{D}(N)$ and $S f=N f$, $f \in \mathcal{D}(S)$.

If a linear operator $S$ in $\mathcal{H}$ is subnormal, then its normal extension $N$ in $\mathcal{K}$ is said to be minimal of spectral type if the only closed subspace of $\mathcal{K}$ reducing $N$ and containing $\mathcal{H}$ is $\mathcal{K}$ itself. Given a normal extension $N$ of $S$, one can always guarantee a minimal normal extension of spectral type (refer to Section 2 of [7]). Let $S$ in $\mathcal{H}$ be subnormal with a normal extension $N$ in $\mathcal{K}$. Let $\mathcal{H}_{s}[N] \equiv$ the closure of $\operatorname{lin}\{E(\sigma) f: f \in \mathcal{H}, \sigma$ is a Borel subset of $\mathcal{C}\}$, where $E(\cdot)$ denotes the spectral measure of $N$. Since $\mathcal{H}_{s}[N]$ reduces $N$, one can define a linear operator $N_{s}$ in $\mathcal{H}_{s}[N]$ by $N_{s} f \equiv N f$ for every $f \in \mathcal{D}\left(N_{s}\right) \equiv \mathcal{D}(N) \cap \mathcal{H}_{s}[N]$. It then follows from Proposition 1 of [7] that $N_{s}$ is a minimal normal extension of $S$ of spectral type. We will refer to $N_{s}$ as the minimal normal extension of $S$ of spectral type associated with the normal extension $N$.

Suppose $S$ is a cyclic operator in $\mathcal{H}$ with $f_{0}$ being a cyclic vector of $S$. As established in Proposition 3 of [6], $S$ is subnormal if and only if there exists a nonnegative measure $\mu$ on the complex plane $\mathcal{C}$ (referred to as the representing measure of $S$ ) such that

$$
\left\langle S^{m} f_{0}, S^{n} f_{0}\right\rangle_{\mathcal{H}}=\int_{\mathcal{C}} z^{m} \bar{z}^{n} d \mu(z) \text { for all } m, n \geq 0 .
$$

If $S$ is a cyclic subnormal operator in $\mathcal{H}$ so that (1.1) is satisfied, then $S$ is unitarily equivalent to $M_{z, \mu}$ in $\mathcal{H}_{\mu}$, where $\mathcal{H}_{\mu}$ is the $L^{2}(\mu)$-closure of the vector space $\mathcal{C}[z]$ of complex polynomials in $z$ and where $M_{z, \mu}$ is the operator of multiplication by $z$ with domain $\mathcal{C}[z]$ (refer to Theorem 5 of [7]); the triple $\left(M_{z, \mu}, \mathcal{C}[z], \mathcal{H}_{\mu}\right)$ will be referred to as a functional model of the cyclic subnormal operator $S$. If $S$ is a cyclic subnormal with the cyclic vector $f_{0}$ and with a minimal normal extension $N$ of spectral type, then it follows from Theorem 5 of [7] that the triple $\left(M_{z, \mu_{N}},\left.\mathcal{C}[z]\right|_{\sigma(N)}, \mathcal{H}_{\mu_{N}}\right)$ is a functional model of $S$, where the positive Borel measure $\mu_{N}(\cdot)$ supported on $\sigma(N)$ is given by $\left\langle E(\cdot) f_{0}, f_{0}\right\rangle$ for the spectral measure $E(\cdot)$ of $N$.

If $S$ is a densely defined closable operator in $\mathcal{H}$, then we use $\bar{S}$ to denote the closure of $S$ in $\mathcal{H}$. It is known that every subnormal operator is closable (refer to 6). Notice that if $S$ is subnormal, then $\bar{S}$ is also subnormal. Let $S$ be a densely defined cyclic subnormal operator in $\mathcal{H}$ with the cyclic vector $f_{0}$. It follows from Proposition 6 of [7] that, for any point $\lambda \in \sigma_{p}\left(S^{*}\right)^{*}$, there exists a unique vector $h_{\lambda}$ in $\mathcal{H}$ such that $p(\lambda)=\left\langle p(S) f_{0}, h_{\lambda}\right\rangle_{\mathcal{H}}$ for every complex polynomial $p$. One can then define a function $k_{S}$ on $\mathcal{C}$ by setting $k_{S}(\lambda)$ equal to $\left\|h_{\lambda}\right\|^{2}$ if $\lambda \in \sigma_{p}\left(S^{*}\right)^{*}$, and equal to $\infty$ otherwise. If $\operatorname{int}\left(\sigma_{p}\left(S^{*}\right)\right)$ is non-empty and if one defines

(1.2) $\gamma(S)=\left\{\lambda \in \mathcal{C}: k_{S}\right.$ is finite and continuous in a neighbourhood of $\left.\lambda\right\}$,

then it follows from Theorem 7 of [7] that $\gamma(S)$ is an open subset of $\operatorname{int}\left(\sigma_{p}\left(S^{*}\right)^{*}\right)$ and that $\operatorname{int}\left(\sigma_{p}\left(S^{*}\right)^{*}\right) \backslash \gamma(S)$ is a nowhere dense subset of $\mathcal{C}$; further, as pointed out in footnote 9 following Theorem 9 of [7], $\sigma(\bar{S}) \backslash \sigma_{a p}(\bar{S}) \subset \gamma(S)$. Given a Hilbert space $\mathcal{H}$ and a cyclic operator $S$ in $\mathcal{H}$ with a cyclic vector $f_{0}$ of $S$, one can come up with a sequence $r=\left\{r_{n}\right\}_{n \geq 0}$ of complex polynomials such that $e=\left\{e_{n}\right\}_{n \geq 0}$ with $e_{n}=r_{n}(S) f_{0}$ an orthonormal basis for $\mathcal{H}$ and such that $\operatorname{lin}\left\{e_{n}: n \geq 0\right\}=$ $\operatorname{lin}\left\{S^{n} f_{0}: n \geq 0\right\}$ (refer to [7]). As observed in the proof of Proposition 6 of [7, the polynomials $r_{n}$ so obtained form a Hamel basis for $\mathcal{C}[z]$, the vector space of complex polynomials in $z$. If $\omega_{r}$ is the set $\left\{z \in \mathcal{C}: \sum_{n=0}^{\infty}\left|r_{n}(z)\right|^{2}<\infty\right\}$, then we 
define $K_{r}(\cdot, \cdot)$ on $\omega_{r} \times \omega_{r}$ by $K_{r}(z, w)=\sum_{n=0}^{\infty} \overline{r_{n}(z)} r_{n}(w) \quad\left(z, w \in \omega_{r}\right)$. Since $K_{r}$ is a positive definite kernel on $\omega_{r}$, we can associate with $K_{r}$ a reproducing kernel Hilbert space $\mathcal{H}_{r}$ as described in [1]. The following theorem is Theorem 6 of [7:

Theorem 1.1. Suppose $\mathcal{H}, S, r, e, \omega_{r}$ and $\mathcal{H}_{r}$ are as described in the preceding paragraph. If the point spectrum $\sigma_{p}\left(S^{*}\right)$ of $S^{*}$ is non-empty, then the following statements are true:

(a) $\mathcal{P}_{r}$, the set of restrictions of members of $\mathcal{C}[z]$ to $\omega_{r}$, is dense in $\mathcal{H}_{r}$;

(b) the operator $M_{z}$ of multiplication by $z$ defined on $\mathcal{P}_{r}$ is cyclic with the cyclic vector the constant polynomial 1 ;

(c) there is a unique partial isometry $W: \mathcal{H} \rightarrow \mathcal{H}_{r}$ with its initial space being the closure of $\operatorname{lin}\left\{\sum_{n=0}^{\infty} \overline{r_{n}(\lambda)} e_{n}: \lambda \in \sigma_{p}\left(S^{*}\right)^{*}\right\}$ and its final space being $\mathcal{H}_{r}$ and such that $W S=M_{z} W$; and

(d) $\omega_{r}=\sigma_{p}\left(S^{*}\right)^{*}=\sigma_{p}\left(M_{z}^{*}\right)^{*}$.

Suppose that, for a cyclic operator $S$ in $\mathcal{H}$ having non-empty point spectrum $\sigma_{p}\left(S^{*}\right), W$ in (c) of Theorem 1.1 turns out to be a unitary of $\mathcal{H}$ onto $\mathcal{H}_{r}$; in this case the triple $\left(M_{z}, \mathcal{P}_{r}, \mathcal{H}_{r}\right)$ will be referred to as an analytic model of the cyclic operator $S$.

1.2. $m$ - $\Sigma$-accretive operators. For a densely defined linear operator $A$ in $\mathcal{H}$ with domain $\mathcal{D}(A)$, let $W(A)$ given by $\{\langle A x, x\rangle: x \in \mathcal{D}(A),\|x\|=1\}$ denote the numerical range of $A$.

Definition 1.2. Let $\Sigma$ denote any open subset of the complex plane. A densely defined linear operator $A$ in $\mathcal{H}$ is said to be $m$ - $\Sigma$-accretive if $\sigma(A) \cap W(A) \subset \bar{\Sigma}$.

Remark 1.3. Let $S$ (resp. $S^{\prime}$ ) be a linear operator in $\mathcal{H}$ (resp. $\mathcal{H}^{\prime}$ ). If there exists a unitary $U$ from $\mathcal{H}$ onto $\mathcal{H}^{\prime}$ such that $U S=S^{\prime} U$ and if $S$ is $m$ - $\Sigma$-accretive, then so is $S^{\prime}$.

For a real $\theta \in\left(0, \frac{\pi}{2}\right)$, let

$$
\begin{aligned}
\Lambda_{\theta} & \equiv\{z \in \mathcal{C}:|\operatorname{Im} z|<\tan \theta \cdot \operatorname{Re} z\}, \\
H_{\theta} & \equiv\{z \in \mathcal{C}:|\operatorname{Im} z|<\theta\} .
\end{aligned}
$$

We remark that the definitions of $m$ - $\Lambda_{\theta}$-accretive and $m$ - $H_{\theta}$-accretive operators as given in Definition 1.2 above and as given in [4 are equivalent. There is a plenteous amount of literature on $m-\Lambda_{\theta}$-accretive and $m$ - $H_{\theta}$-accretive operators. See, for example, the recent monograph [4] and the references cited therein.

We conclude this section with the following useful observation.

Lemma 1.4. Let $S$ be a subnormal operator in $\mathcal{H}$ with a normal extension $N$ in $\mathcal{K}$ and let $\theta \in\left(0, \frac{\pi}{2}\right)$. Let $\Lambda_{\theta}$ and $H_{\theta}$ be as in (1.3) and (1.4) respectively. If $\sigma(N) \subset \overline{\Lambda_{\theta}}$ $\left(\right.$ resp. $\left.\overline{H_{\theta}}\right)$, then $W(S) \subset \overline{\Lambda_{\theta}}\left(\right.$ resp. $\left.\overline{H_{\theta}}\right)$.

Proof. The desired inclusions can be easily deduced using the Spectral Theorem for unbounded normal operators. To check that let $E(\cdot)$ denote the spectral measure 
of $N$. Let $x \in \mathcal{D}(S)$ be such that $\|x\|_{\mathcal{H}}=1$. Then

$$
\begin{aligned}
\left|\operatorname{Im}\langle S x, x\rangle_{\mathcal{H}}\right| & =\left|\operatorname{Im}\langle N x, x\rangle_{\mathcal{K}}\right| \\
& =\left|\operatorname{Im} \int_{\sigma(N)} z d\langle E(z) x, x\rangle_{\mathcal{K}}\right| \\
& \leq \int_{\sigma(N)}|\operatorname{Im} z| d\langle E(z) x, x\rangle_{\mathcal{K}} .
\end{aligned}
$$

It is now easy to check that $\langle S x, x\rangle_{\mathcal{H}}$ belongs to $\overline{\Lambda_{\theta}}$ (resp. $\overline{H_{\theta}}$ ) whenever $\sigma(N) \subset \overline{\Lambda_{\theta}}$ (resp. $\overline{H_{\theta}}$ ).

\section{Bounded $H^{\infty}$ FUnCTIONAL CALCULI FOR UNBOUNDED SUBNORMALS}

The key observation in this paper is the following Spectral Exclusion Principle for unbounded subnormals (cf. ([3, Lemma 1) and ([2, Lemma 2.2)).

Lemma 2.1. Let $S$ in $\mathcal{H}$ be cyclic with the cyclic vector $f_{0}$, and subnormal with a normal extension $N$. Let $N_{s}$ be the minimal normal extension of $S$ of spectral type associated with $N$. Let

$$
\begin{aligned}
& H_{r}^{1} \equiv\{\mu \in \mathcal{C}: \operatorname{Re} \mu<r\}, H_{r}^{2} \equiv\{\mu \in \mathcal{C}: \operatorname{Re} \mu>r\}, \\
& H_{r}^{3} \equiv\{\mu \in \mathcal{C}: \operatorname{Im} \mu<r\}, H_{r}^{4} \equiv\{\mu \in \mathcal{C}: \operatorname{Im} \mu>r\},
\end{aligned}
$$

for some real $r$. Let $\alpha_{1}=-1, \alpha_{2}=1, \alpha_{3}=i$, and $\alpha_{4}=-i$. Let $\Omega$ stand for $H_{r}^{j}$ and let $\alpha$ stand for $\alpha_{j}(j=1,2,3,4)$. Suppose that $S$ admits an analytic model and that $\sigma(N) \subset \Omega^{c}$. Let $\omega$ be the unbounded connected component of $\sigma(N)^{c}$ in $\mathcal{C}$ that contains $\Omega$. If $\left\{\sum_{n=0}^{k} \frac{(\alpha m)^{n} S^{n} f_{0}}{n !}\right\}_{k \geq 0}$ converges in $\mathcal{H}$ for every integer $m \geq 1$, then the following statements are true.

(1) $\omega$ is contained in $\sigma(\bar{S})^{c}$.

(2) If in addition $\sigma(N)^{c}$ is connected, then $\sigma(\bar{S})=\sigma\left(N_{s}\right)$.

Proof. Assume the hypotheses. Assume also that $\left\{\sum_{n=0}^{k} \frac{(\alpha m)^{n} S^{n} f_{0}}{n !}\right\}_{k \geq 0}$ converges in $\mathcal{H}$ for every integer $m \geq 1$. The proof here is an adaption of the argument of ([3], Lemma 1); see also ([2], Lemma 2.2). Suppose that $\omega$ is not contained in $\sigma(\bar{S})^{c}$, where $\omega$ is the unbounded connected component of $\sigma(N)^{c}$ that contains $\Omega$. From the proof of Theorem 2 of [7] (refer to Remark 1 of [3]) one has either $\omega \cap \sigma(\bar{S})=\emptyset$ or $\omega \subset \sigma(\bar{S})$. Since $\omega$ is not contained in $\sigma(\bar{S})^{c}$, one has $\omega \subset \sigma(\bar{S})$.

With $\gamma(S)$ as defined in (1.2), $\sigma(\bar{S}) \backslash \sigma_{a p}(\bar{S}) \subset \gamma(S)$, as recorded in our comments following (1.2). Since $N$ extends $\bar{S}$, we have $\sigma_{a p}(\bar{S}) \subset \sigma_{a p}(N)=\sigma(N)$ and thus $\sigma(\bar{S}) \backslash \sigma(N) \subset \gamma(S)$. Since $\omega \subset \sigma(\bar{S})$ and $\omega \cap \sigma(N)=\emptyset$, we have $\omega \subset \gamma(S)$. From the observations following (1.2) in Section 1, we have $\gamma(S) \subset \operatorname{int}\left(\sigma_{p}\left(S^{*}\right)^{*}\right)$. By hypothesis, $\Omega \subset \omega$, so that $\Omega \subset \sigma_{p}\left(S^{*}\right)^{*}$.

Let $h \in \Omega$ and let $\epsilon>0$ be such that $h+\bar{\alpha} \epsilon \in \Omega$. Consider the sequence $\left\{g_{k} \equiv \sum_{n=0}^{k} \exp \{n(\alpha z-\alpha h-\epsilon)\}\right\}_{k \geq 0}$. We claim that $\left\{\left.g_{k}\right|_{\sigma_{p}\left(S^{*}\right)^{*}}\right\}_{k \geq 0}$ is convergent in $\mathcal{H}_{r}$. Let $N_{s}$ be the minimal normal extension of $S$ of spectral type associated with $N$. Then $S$ admits the functional model $\left(M_{z, \mu_{N_{s}}},\left.\mathbb{C}[z]\right|_{\sigma\left(N_{s}\right)}, \mathcal{H}_{\mu_{N_{s}}}\right)$ (refer to the discussion following (1.1)). We check that $\left\|f-\left.g_{m}\right|_{\sigma\left(N_{s}\right)}\right\|_{\mathcal{L}}$ converges to 0 as $m$ tends to $\infty$ for some $f$ in $\mathcal{L}=L^{2}\left(\sigma\left(N_{s}\right), \mu_{N_{s}}\right)$. Since $N_{s}$ is a minimal normal extension of spectral type of $S$, by Theorem 5 of [7, one has $\operatorname{supp}\left(\mu_{N_{s}}\right)=\sigma\left(N_{s}\right)$. 
Also, since $\mathcal{D}\left(N_{s}\right) \subset \mathcal{D}(N)$ and since $N x=N_{s} x\left(x \in \mathcal{D}\left(N_{s}\right)\right)$, it follows that $\sigma\left(N_{s}\right)=\sigma_{a p}\left(N_{s}\right) \subset \sigma_{a p}(N)=\sigma(N)$. Hence $\operatorname{supp}\left(\mu_{N_{s}}\right) \subset \sigma(N)$. Note that for any integer $m \geq 0$ and for any $z \in \sigma(N) \subset \Omega^{c}$, one has

$$
\begin{aligned}
\left|\sum_{k \geq m+1} \exp \{(\alpha z-\alpha h-\epsilon) k\}\right| & \leq \sum_{k \geq m+1}|\exp \{(\alpha z-\alpha h-\epsilon) k\}| \\
& \leq \sum_{k \geq m+1} \exp (-\epsilon k)
\end{aligned}
$$

and the last expression tends to 0 as $m$ tends to $\infty$. In view of $\operatorname{supp}\left(\mu_{N_{s}}\right) \subset \sigma(N)$, one has

$$
\begin{aligned}
\left\|\sum_{k \geq m+1} \exp \{(\alpha z-\alpha h-\epsilon) k\}\right\|_{\mathcal{L}}^{2} & =\int\left|\sum_{k \geq m+1} \exp \{(\alpha z-\alpha h-\epsilon) k\}\right|^{2} d \mu_{N_{s}}(z) \\
& =\int_{\sigma(N)}\left|\sum_{k \geq m+1} \exp \{(\alpha z-\alpha h-\epsilon) k\}\right|^{2} d \mu_{N_{s}}(z) \\
& \leq\left(\sum_{k \geq m+1} \exp (-\epsilon k)\right)^{2} \mu_{N_{s}}(\sigma(N)) .
\end{aligned}
$$

The preceding arguments show that for some $f \in \mathcal{L},\left\|f-\left.g_{m}\right|_{\sigma\left(N_{s}\right)}\right\|_{\mathcal{L}}$ converges to 0 as $m$ tends to $\infty$. By assumption, one has for every integer $k \geq 1,\left.g_{m}\right|_{\sigma\left(N_{s}\right)} \in \mathcal{H}_{\mu_{N_{s}}}$. It is now clear that $f \in \mathcal{H}_{\mu_{N_{s}}}$. Since $S$ admits an analytic model as well as a functional model, there exists a unique unitary $V: \mathcal{H}_{\mu_{N_{s}}} \rightarrow \mathcal{H}_{r}$, where $\left(M_{z}, \mathcal{P}_{r}, \mathcal{H}_{r}\right)$ stands for the analytic model for $S$. Thus $g \equiv V f$ is such that $\left\|g-\left.g_{k}\right|_{\sigma_{p}\left(S^{*}\right)^{*}}\right\|_{\mathcal{H}_{r}}$ converges to 0 as $m$ tends to $\infty$. Thus the claim stands verified.

Since $\Omega \subset \sigma_{p}\left(S^{*}\right)^{*}$ and since $h+\bar{\alpha} \epsilon \in \Omega$, one has $h+\bar{\alpha} \epsilon \in \sigma_{p}\left(S^{*}\right)^{*}$. By Lemma 2 of [7, there exists a constant $c_{h}>0$ such that

$$
|p(h+\bar{\alpha} \epsilon)| \leq c_{h}\left\|p(S) f_{0}\right\|_{\mathcal{H}} \text { for every polynomial } p .
$$

Since $S$ admits the analytic model $\left(M_{z}, \mathcal{P}_{r}, \mathcal{H}_{r}\right)$ and $\left.g_{k}\right|_{\sigma_{p}\left(S^{*}\right)^{*}} \in \mathcal{H}_{r}$, by Part (a) of Theorem 1.1, there exists a sequence of complex polynomials $\left\{p_{n, k}\right\}$ such that $\left\|p_{n, k}-\left.g_{k}\right|_{\sigma_{p}\left(S^{*}\right)^{*}}\right\|_{\mathcal{H}_{r}} \rightarrow 0$ as $n$ tends to $\infty$ for every integer $k \geq 1$. Since $h+\bar{\alpha} \epsilon \in \sigma_{p}\left(S^{*}\right)^{*}$ and since $\mathcal{H}_{r}$ is a reproducing kernel Hilbert space, it follows that $\left|p_{n, k}(h+\bar{\alpha} \epsilon)-(k+1)\right|$ converges to 0 as $n$ tends to $\infty$. In view of (2.4) one has

$$
\left|p_{n, k}(h+\bar{\alpha} \epsilon)\right| \leq c_{h}\left\|p_{n, k}(S) f_{0}\right\|_{\mathcal{H}}=\left\|p_{n, k}\right\|_{\mathcal{H}_{r}} .
$$

The passage of $n$ to $\infty$ in the preceding inequality yields $k+1 \leq c_{h}\left\|\left.g_{k}\right|_{\sigma_{p}\left(S^{*}\right)^{*}}\right\|_{\mathcal{H}_{r}}$. This contradicts the fact that $\left\{\left\|\left.g_{k}\right|_{\sigma_{p}\left(S^{*}\right)^{*}}\right\|\right\}_{k \geq 1}$ converges to $\|g\|_{\mathcal{H}_{r}}$ as $k$ tends to $\infty$. Thus we must have $\omega \subset \sigma(\bar{S})^{c}$.

Now assume in addition that $\sigma(N)^{c}$ is connected so that $\omega^{c}=\sigma(N)$. From what we saw above, for any normal extension $N$ of $S$, one has $\sigma(\bar{S}) \subset \sigma(N)$. In particular, one has $\sigma(\bar{S}) \subset \sigma\left(N_{s}\right)$, where $N_{s}$ is the minimal normal extension of $S$ of spectral type associated with $N$. Since $N_{s}$ is a normal extension of spectral type of $\bar{S}$, by the Spectral Inclusion Theorem for unbounded subnormals ([7], Theorem 1), $\sigma\left(N_{s}\right) \subset \sigma(\bar{S})$. Hence $\sigma(\bar{S})=\sigma\left(N_{s}\right)$ as desired. 
Theorem 2.2. Let $S$ in $\mathcal{H}$ be cyclic with the cyclic vector $f_{0}$, and subnormal with a normal extension $N$. For $\theta \in\left(0, \frac{\pi}{2}\right)$, let

$$
\Sigma_{\theta}^{1} \equiv \Lambda_{\theta}, \Sigma_{\theta}^{2} \equiv H_{\theta}
$$

(see (1.3) and (1.4)). Let $S_{1}=\{-1\}, S_{2}=\{i,-i\}$, and fix $j \in\{1,2\}$. Suppose that $S$ admits an analytic model and that $\sigma(N) \subset \overline{\Sigma_{\theta}^{j}}$. If $\left\{\sum_{n=0}^{k} \frac{(\alpha m)^{n} S^{n} f_{0}}{n !}\right\}_{k \geq 0}$ converges in $\mathcal{H}$ for every integer $m \geq 1$ and for every $\alpha \in S_{j}$, then $\bar{S}$ is $m-\Sigma_{\theta^{j}}$. accretive.

Proof. This is immediate from Lemma 1.4 and (1) of the preceding lemma.

For an open subset $\Omega$ of the complex plane, let $H^{\infty}(\Omega)$ stand for

$$
\{f: \Omega \rightarrow \mathcal{C}: f \text { is a bounded holomorphic function }\} .
$$

The vector space $H^{\infty}(\Omega)$ is a normed linear space endowed with the supremum norm $\|f\|_{\infty, \Omega} \equiv \sup _{z \in \Omega}|f(z)|$.

Corollary 2.3. Let $S, \mathcal{H}, f_{0}, N, \Sigma_{\theta}^{j}, \mathcal{H}_{r}$ be as in the previous theorem. Suppose that $\sigma_{p}\left(S^{*}\right)^{*}$ has non-empty interior and let $\left(M_{z, \mu}, \mathbb{C}[z], \mathcal{H}_{\mu}\right)$ denote a functional model of $S$. Then the following statements are true.

(1) ([2], Proposition 3.4) If $\sigma(N) \subset \overline{\Sigma_{\omega}^{1}}\left(\omega \in\left(0, \frac{\pi}{2}\right)\right)$ and $\left\{\sum_{n=0}^{k} \frac{(-m)^{n} z^{n}}{n !}\right\}_{k \geq 0}$ converges in $\mathcal{H}_{\mu}$ for every integer $m \geq 1$, then for every $\theta \in\left(\omega, \frac{\pi}{2}\right)$,

$$
\left\|f\left(\overline{M_{z, \mu}}\right)\right\| \leq\left(2+\frac{2}{\sqrt{3}}\right)\|f\|_{\infty, \Lambda_{\theta}}\left(f \in H^{\infty}\left(\Lambda_{\theta}\right)\right) .
$$

(2) If $\sigma(N) \subset \overline{\Sigma_{\omega}^{2}}\left(\omega \in\left(0, \frac{\pi}{2}\right)\right)$, and the sequences $\left\{\sum_{n=0}^{k} \frac{(i m)^{n} z^{n}}{n !}\right\}_{k \geq 0}$ and $\left\{\sum_{n=0}^{k} \frac{(-i m)^{n} z^{n} f_{0}}{n !}\right\}_{k \geq 0}$ converge in $\mathcal{H}_{\mu}$ for every integer $m \geq 1$, then for every $\theta \in\left(\omega, \frac{\pi}{2}\right)$,

$$
\left\|f\left(\overline{M_{z, \mu}}\right)\right\| \leq\left(2+\frac{2}{\sqrt{3}}\right)\|f\|_{\infty, H_{\theta}}\left(f \in H^{\infty}\left(H_{\theta}\right)\right) .
$$

Proof. For a proof of (1), the reader is referred to (2], Proof of Proposition 3.4). Since there exists a unitary $U$ from $\mathcal{H}$ onto $\mathcal{H}_{\mu}$ such that $U S=M_{z, \mu} U$, one has $U \bar{S}=\overline{M_{z, \mu}} U$. Hence by Remark 1.3 and the previous theorem, $\overline{M_{z, \mu}}$ is $m-\Sigma_{\theta^{-}}^{j}$ accretive. The desired estimate in (2) follows from Theorem 7.4.7 of [4].

\subsection{Applications: Polynomial approximation results in the functional model spaces.}

Proposition 2.4. Let $S$ in $\mathcal{H}$ be cyclic with the cyclic vector $f_{0}$, and subnormal with a normal extension $N$. Let $\left(M_{z, \mu}, \mathbb{C}[z], \mathcal{H}_{\mu}\right)$ denote a functional model of $S$. For $\omega \in\left(0, \frac{\pi}{2}\right)$, let

$$
\begin{gathered}
\Sigma_{\omega}^{1} \equiv \Lambda_{\omega}, \quad \Sigma_{\omega}^{2} \equiv H_{\omega} ; \\
X_{\omega}^{1}=H^{\infty}\left(\Lambda_{\omega}\right), \quad X_{\omega}^{2}=H^{\infty}\left(H_{\omega}\right) .
\end{gathered}
$$

Let $S_{1}=\{-1\}, S_{2}=\{i,-i\}$ and fix $j \in\{1,2\}$. Suppose that $S$ admits an analytic model and that $\sigma(N) \subset \overline{\Sigma_{\omega}^{j}}$. If $\left\{\sum_{n=0}^{k} \frac{(\alpha m)^{n} z^{n}}{n !}\right\}_{k \geq 0}$ converges in $\mathcal{H}_{\mu}$ for every 
integer $m \geq 1$ and for every $\alpha \in S_{j}$, then $X_{\theta}^{j}$ is contained in the functional model space $\mathcal{H}_{\mu}$ for every $\theta \in\left(\omega, \frac{\pi}{2}\right)$.

Proof. Since the case $j=1$ is already treated in (2], Corollary 3.5), we deal here only with $j=2$. In view of Corollary 2.3 , it suffices to verify that the action of $f\left(\overline{M_{z, \mu}}\right)$ is multiplication by $f$. Since $\sigma\left(\overline{M_{z, \mu}}\right) \subset \overline{\Sigma_{\omega}^{j}}$, it follows that the action of $(z-\mu)^{-1}\left(\overline{M_{z, \mu}}\right) \equiv\left(\overline{M_{z, \mu}}-\mu\right)^{-1}$ is multiplication by $(z-\mu)^{-1}$ for all $\mu \notin \overline{\Lambda_{\theta}}$. Thus for every rational function $f$ with poles lying outside $\overline{\Lambda_{\theta}}$ the action of $f\left(\overline{M_{z, \mu}}\right)$ is multiplication by $f$ (see Proposition F.3. of [4]).

Let $f \in X_{\theta}^{j}$. By Proposition F.4 of [4] there exists a sequence of rational functions with poles lying outside $\overline{\Sigma_{\theta}^{j}}$ such that $\left\|r_{n}\right\|_{\Sigma_{\theta}^{j}, \infty} \leq\|f\|_{\Sigma_{\theta}^{j}, \infty}$ for all $n$, and $r_{n} \rightarrow f$ pointwise on $\Sigma_{\theta}^{j}$. In particular, $\sup _{n \geq 0}\left\|r_{n}\right\|_{\infty, \Sigma_{\theta}^{j}}<\infty$. By the preceding corollary, one has

$$
\sup _{n \geq 0}\left\|r_{n}\left(\overline{M_{z, \mu}}\right)\right\|_{\mathcal{H}_{\mu}} \leq\left(2+\frac{2}{\sqrt{3}}\right) \sup _{n \geq 0}\left\|r_{n}\right\|_{\infty, \Sigma_{\theta}^{j}}<\infty
$$

Now one may appeal to the Convergence Lemma (4], Proposition 5.1.7(b)) to conclude that $\left\|r_{n}\left(\overline{M_{z, \mu}}\right) x-f\left(\overline{M_{z, \mu}}\right) x\right\|_{\mathcal{H}_{\mu}} \rightarrow 0$ for every $x \in \mathcal{H}_{\mu}$. Since the action of each $r_{n}\left(\overline{M_{z, \mu}}\right)$ is multiplication by $r_{n}$, a standard measure-theoretic argument shows that the action of $f\left(\overline{M_{z, \mu}}\right)$ is multiplication by $f$. This completes the proof of the corollary.

Corollary 2.5. Assume the hypotheses of the previous proposition. Assume also that $\left\{\sum_{n=0}^{k} \frac{(\alpha m)^{n} z^{n}}{n !}\right\}_{k \geq 0}$ converges in $\mathcal{H}_{\mu}$ for every integer $m \geq 1$ and for every $\alpha \in S_{j}$. For every $\theta \in\left(\omega, \frac{\pi}{2}\right)$ and for every $f$ in $X_{\theta}^{j}$ there exists a sequence $\left\{p_{n}\right\}_{n \geq 0}$ of complex polynomials such that $\int_{\Sigma_{\omega}^{j}}\left|p_{n}(z)-f(z)\right|^{2} d \mu(z)$ converges to 0 as $n \rightarrow \infty$.

The essence of the present paper is the following example.

Example 2.6. Fix an $\omega \in\left(0, \frac{\pi}{2}\right)$. Let $\Sigma_{\omega}^{1} \equiv \Lambda_{\omega}, \Sigma_{\omega}^{2} \equiv H_{\omega}$ and let $X_{\omega}^{1}=$ $H^{\infty}\left(\Lambda_{\omega}\right), X_{\omega}^{2}=H^{\infty}\left(H_{\omega}\right)$. Let $S_{1}=\{-1\}, S_{2}=\{i,-i\}$ and fix $j \in\{1,2\}$. Let $\theta \in\left(\omega, \frac{\pi}{2}\right)$ and let $f$ be in $X_{\theta}^{j}$.

Let $\mathcal{H}_{j}$ be the closure of the complex vector space of all complex polynomials $\mathcal{C}[z]$ in $\mathcal{K}_{j} \equiv L^{2}\left(\Sigma_{\omega}^{j}, \exp \left(-|z|^{2}\right) d z\right)$. Consider the operator $T_{j}$ of multiplication by the coordinate function $z$ in $\mathcal{H}_{j}$ with domain $\mathcal{C}[z]$. Then $T_{j}$ is a cyclic operator with the cyclic vector $1_{j}$, where $1_{j}(z)=1\left(z \in \Sigma_{\omega}^{j}\right)$. If $N_{j}$ denotes the operator of multiplication by $z$ in $\mathcal{K}_{j}$ with domain $\left\{f \in \mathcal{K}_{j}: N_{j} f \in \mathcal{K}_{j}\right\}$, then $N_{j}$ is a normal extension of $T_{j}$ with $\sigma\left(N_{j}\right)=\overline{\Sigma_{\omega}^{j}}$. The fact that $T_{j}$ admits an analytic model can be deduced from Lemma 4.2 of [2]. Also, it is easy to see that $\left\{\sum_{n=0}^{k} \frac{(\alpha m)^{n} T_{j}^{n} f_{0}}{n !}\right\}_{k>0}$ converges in $\mathcal{H}_{j}$ for every integer $m \geq 1$ and for every $\alpha \in S_{j}$. By the preceding corollary, there exists a sequence $\left\{p_{n}^{j}\right\}_{n \geq 0}$ of complex polynomials such that

$$
\lim _{n \rightarrow \infty} \int_{\Sigma_{\omega}^{j}}\left|p_{n}^{j}(z)-f(z)\right|^{2} \exp \left(-|z|^{2}\right) d z=0 .
$$




\section{REFERENCES}

[1] N. Aronszajn, Theory of reproducing kernels, Trans. Amer. Math. Soc. 68 (1950), 337-404. MR0051437 (14:479c)

[2] S. Chavan, On a Friedrichs extension related to unbounded subnormals. II, Glasgow Math. J. 50 (2008), 97-109.

[3] S. Chavan and A. Athavale, On a Friedrichs extension related to unbounded subnormals. I, Glasgow Math. J. 48 (2006), 19-28. MR2224923 (2007b:47049)

[4] M. Haase, The Functional Calculus for Sectorial Operators, Operator Theory: Advances and Applications, Vol. 169, Birkhäuser, 2006. MR2244037 (2007j:47030)

[5] M. Miklavčič, Applied Functional Analysis and Partial Differential Equations, World Scientific, River Edge, NJ, 1998. MR.1784426 (2001k:47001)

[6] J. Stochel and F. Szafraniec, On normal extensions of unbounded operators. II, Acta Sci. Math. (Szeged) 53 (1989), 153-177. MR1018684(91i:47032)

[7] J. Stochel and F. Szafraniec, On normal extensions of unbounded operators. III, Spectral properties, Publ. RIMS, Kyoto Univ. 25 (1989), 105-139. MR999353 (91i:47033)

Indian Institute of Science Education and Research Pune, Pune-411008, India

E-mail address: sl.chavan@iiserpune.ac.in

Current address: Harish-Chandra Research Institute, Chhatnag Road, Jhusi, Allahabad, 211019 India

E-mail address: chavansameer@hri.res.in 